\title{
Parâmetros genéticos para produção de leite no dia do controle de vacas da raça Holandesa utilizando modelos de análises de fatores e componentes principais
}

\author{
Genetic parameters for test day milk production of Holstein cows using \\ factors and principal components analysis
}

\author{
Mariana de Almeida Dornelles ${ }^{\mathrm{I}^{*}}$ Paulo Roberto Nogara Rorato $^{\mathrm{I}}$ Fernanda Cristina Breda $^{\mathrm{I}}$ \\ Carlos Bondan" Luis Telo Lavadinho da Gama ${ }^{\text {III }}$ Jaime Araujo Cobuci ${ }^{\text {IV }}$ \\ Giovani Luis Feltes ${ }^{I}$ Vanessa Tomazetti Michelotti ${ }^{I}$ Alan Miranda Prestes ${ }^{I}$
}

RESUMO

Objetivou-se comparar um modelo multicaracterística padrão com modelos de análise de fatores $(A F)$ e de componentes principais $(C P)$ para estimar parâmetros genéticos para a produção de leite no dia do controle (PLDC) de vacas da raça Holandesa. $O$ arquivo de trabalho constituiu-se de 4.616 registros mensais de PLDC de primeiras lactações de vacas da raça Holandesa. As PLDC foram agrupadas em dez classes mensais, entre o $5^{\circ}$ e $305^{\circ}$ dia da lactação (PLDC1 a PLDC10). Foram realizadas análises considerando 11 modelos diferentes, como segue: multi-característica padrão (MC); cinco modelos de posto reduzido, para a matriz de covariância genética, ajustando um a cinco (CP1 ... CP5) componentes principais; e dois modelos utilizando análise de fatores (F1, F2, F3, F4 e F5). Para todos os modelos, foram considerados como aleatórios os efeitos genético aditivo e o residual e como fixos os de grupo de contemporâneos, da idade da vaca ao parto (linear e quadrático) e dias em lactação (linear). Os valores de $\log L$, AIC e BIC melhoraram com o aumento do número de parâmetros até CP4 e AF4. Comparando CP4 e AF4, observa-se que CP4 resultou em melhores valores de $\log L$, AIC e BIC. As estimativas de herdabilidade e correlações genéticas utilizando os modelos MC, CP4 e AF4 foram similares, variando de 0,06 (PL6) a 0,65 (PL10) e de 0,05 (PL4xPL10) a 0,94 (PL2xPL3), respectivamente, indicando que a estrutura de covariâncias genéticas entre as produções de leite no dia do controle pode ser ajustada utilizando um modelo de posto reduzido, contendo quatro componentes principais ou quatro fatores.

Palavras-chave: componentes de variância, correlações genéticas, herdabilidade, posto reduzido.

\section{ABSTRACT}

The objective was to compare a standard multi-trait (MT) analysis model with factor (FA) and principal components
(PC) analyses models to estimated genetic parameters for Holstein cows test day milk production (TD). The data file was composed by 4.616 TD at first lactation registers. The TD was grouped into ten monthly classes of lactation, from the 5th and the 305th day of lactation (TD1 to TD10). Analyses were performed considering 11 different models: standard multi-traits (MT), five reduced rank models to genetic covariance matrix adjusting one (PC1), two (PC2), three (PC3), four (PC4) and five (PD5) principal components and five models using factor analyses (F1, F2, F3, F4 and F5). To all the models the effects additive genetic and residual were considered as random and the effects of contemporary group, age of cow at parturition (linear and quadratic) and days in lactation (linear) were considered as fixed. The values of $\log L, A I C$ e BIC improved with the augment of the number of parameters until CP4 and AF4. Comparing CP4 and AF4 is possible to verify that CP4 proportioned better values to $\log L$, AIC e BIC. The heritabilities and genetic correlations estimated to the ten test day milk production using MC, CP4 and AF4 models were similar ranging from 0.06 (PL6) to 0.65 (PL10) and from 0.05 (PL4xPL10) to 0.94 (PL2xPL3), respectively, indicating that the structure of the genetic covariance between the TD milk productions can be adjusted using a reduced rank model with four principal components or four factors.

Key words: components of variance, genetic correlation, heritability, reduced rank.

\section{INTRODUÇÃO}

A utilização da produção de leite no dia do controle (PLDC) em substituição à produção acumulada em até 305 dias tem sido proposta como critério de seleção. A utilização de test-day

'Departamento de Zootecnia, Centro de Ciências Rurais (CCR), Universidade Federal de Santa Maria (UFSM), Avenida Roraima, 1000, Cidade Universitária, Camobi, 97105-900, Santa Maria, RS, Basil. E-mail: marizootecnia@hotmail.com. *Autor para correspondência.

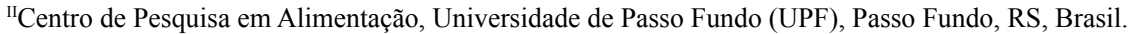

IIIServiço de Análise de Rebanhos Leiteiros, Universidade Técnica de Lisboa, Porto, Portugal.

${ }^{\text {IV }}$ Departamento de Zootecnia, Universidade Federal do Rio Grande do Sul (UFRGS), Porto Alegre, RS, Brasil. Recebido 18.07.14 Aprovado 31.10.14 Devolvido pelo autor 20.02.15 CR-2014-1076.R1 
models (TDM) possibilita a inclusão de lactações incompletas, sem a necessidade de projeções, permitindo a realização de avaliações mais frequentes e, consequentemente, reduzindo o intervalo de geração (SWALVE, 1995). Entretanto, segundo BIGNARDI et al. (2010), a maior desvantagem dos TDM é a dificuldade computacional de analisar um elevado volume de dados, além da necessidade de estimar um número maior de parâmetros genéticos, uma vez que, utilizando modelo multi-característica completo, cada PLDC é considerada como uma característica distinta, aumentando linearmente o número de efeitos no modelo, de acordo com o número de controles considerados.

Com a finalidade de diminuir a demanda computacional no processo de estimação para avaliação de múltiplas características, as metodologias de componentes principais e análise de fatores têm sido propostas. Os primeiros a utilizarem a matriz de covariância com ranking reduzido para avaliação de múltiplas características entre países foram MADSEN et al. (2000). O método consistia em remover autovalores negativos, ou seja, não era um método real de redução de ranking. Posteriormente, THOMPSON et al. (2003) e KIRKPATRICK \& MEYER (2004) sugeriram a estimação direta dos fatores e componentes principais, respectivamente.

Ambas as análises de fatores e de componentes principais têm como principal objetivo a redução da dimensionalidade. As diferenças entre as duas técnicas podem ser colocadas da seguinte maneira: na análise de fatores, as variáveis são expressas como combinações lineares dos fatores, enquanto que os componentes principais são funções lineares das variáveis; na análise de componentes principais, a ênfase é explicar a variância total; e, na análise de fatores, o objetivo é tentar explicar as covariâncias. A análise de componentes principais não requer pressuposições, enquanto que a análise de fatores requer várias pressuposições importantes ou fundamentais; os componentes principais são únicos, enquanto que os fatores estão sujeitos a uma rotação arbitrária e, se o número de fatores for alterado, os fatores também se alteram.

O objetivo neste trabalho foi comparar um modelo multi-característica padrão com modelos de análises de fatores e componentes principais para estimar parâmetros genéticos para a produção de leite no dia do controle de vacas da raça Holandesa

\section{MATERIAL E MÉTODOS}

Os dados fornecidos pelo Serviço de Análises de Rebanhos Leiteiros da Universidade de Passo Fundo (SARLE/UPF) foram coletados entre os anos de 2007 e 2011. As produções de leite no dia do controle (PLDC) foram agrupadas em dez classes mensais de lactação, obtidas entre cinco e 305 dias após o parto (PLDC1 a PLDC10). O grupo de contemporâneas (GC) para as PLDC foi definido como rebanho-ano-estação do controle (1ª estação: janeiro-março, 2 ${ }^{\text {a }}$ estação: abril a junho, $3^{\text {a }}$ estação: julho a setembro e 4a estação: outubro a dezembro). Foram excluídas das análises as PLDC com mais ou menos três desvios padrão em relação à média dentro de cada GC e vacas com idade ao parto inferior a $22 \mathrm{e}$ superior a 48 meses. $\mathrm{O}$ arquivo de trabalho constituiuse de 4.616 registros mensais de PLDC provenientes de primeiras lactações de vacas da raça Holandesa, provenientes de 46 rebanhos (Tabela 1).

Foram realizadas análises considerando 11 diferentes modelos, como segue: multi-característica padrão (MC); cinco modelos de posto reduzido, para a matriz de covariância genética, ajustando um (CP1),

Tabela 1 - Número de controles (N), média e desvio-padrão (DP), período em dias e número de animais por grupo de contemporâneas (GC) para as características de produção de leite ao longo da lactação.

\begin{tabular}{|c|c|c|c|c|c|}
\hline Característica & № & Média $(\mathrm{kg})$ & $\mathrm{DP}(\mathrm{kg})$ & Período (dias) & GC \\
\hline PLDC1 & 430 & 27,78 & 8,38 & $6-30$ & 65 \\
\hline PLDC2 & 686 & 30,49 & 8,19 & $31-60$ & 102 \\
\hline PLDC3 & 691 & 30,88 & 7,97 & $61-90$ & 104 \\
\hline PLDC4 & 613 & 30,62 & 8,01 & $91-120$ & 93 \\
\hline PLDC5 & 565 & 29,60 & 8,16 & $121-150$ & 85 \\
\hline PLDC6 & 472 & 28,44 & 7,68 & $151-180$ & 79 \\
\hline PLDC7 & 393 & 28,14 & 7,78 & $181-210$ & 65 \\
\hline PLDC8 & 310 & 27,53 & 7,08 & $211-240$ & 53 \\
\hline PLDC9 & 266 & 26,41 & 7,78 & $241-270$ & 47 \\
\hline PLDC10 & 190 & 26,12 & 7,07 & 271-305 & 35 \\
\hline
\end{tabular}


dois (CP2), três (CP3), quatro (CP4) e cinco (CP5) componentes principais; e cinco modelos utilizando análise de fatores (F1, F2, F3, F4 e F5). Em todos os modelos, foram considerados os efeitos aleatórios genético aditivo e residual. Foram considerados os efeitos fixos de grupo de contemporâneos, idade da vaca ao parto (linear e quadrático) e dias em lactação (linear).

Para a análise envolvendo o modelo $\mathrm{MC}$, foi utilizado o modelo linear misto: $\mathrm{y}=\mathrm{Xb}+\mathrm{Zu}+e$, em que: y é o vetor de observações para as produções de leite no dia do controle (PLDC1 a PLDC10); b vetor de soluções para os efeitos fixos; $\mathbf{u}=$ vetor de soluções para os efeitos aleatórios genéticos aditivos; $\mathrm{X}$ e $\mathrm{Z}$ matrizes de incidência relacionando $\mathrm{b}$ e $\mathrm{u}$, respectivamente, e $\boldsymbol{e}$ é o vetor do efeito aleatório residual.

Para as análises de componentes principais, o modelo MC foi reparametrizado, produzindo um modelo equivalente: $\mathrm{y}=\mathrm{Xb}+\mathrm{Z}^{*} \mathrm{u}^{*}+e$, em que: $\mathrm{Z}^{*}=\mathrm{Z}\left(\mathrm{I}_{\mathrm{NA}} \ddot{\mathrm{A}} \mathrm{Q}_{\mathrm{m}}\right)$ e $\mathrm{u}^{*}=\left(\mathrm{I}_{\mathrm{NA}} \ddot{A}^{\prime}{ }_{\mathrm{m}}\right) \mathrm{u}, \mathrm{com} \operatorname{Var}\left(\mathrm{u}^{*}\right)=\mathrm{A} \ddot{\mathrm{A}} \mathrm{I}_{\mathrm{m}}$ e $\mathrm{Q}_{\mathrm{m}}=\mathrm{E}_{\mathrm{m}}\left(\mathrm{L}_{\mathrm{m}}\right)^{1 / 2}$, em que $\mathrm{N}_{\mathrm{A}}$ é o número de animais incluído na análise; $\mathrm{m}$ é o número de componentes principais ajustados; E é a matriz $(\mathrm{K} \times \mathrm{m})$ dos primeiros $\mathrm{m}$ autovetores da matriz de (co)variâncias para o efeito genético aditivo $\left(\mathrm{S}_{\mathrm{A}}\right)$, $\mathrm{k}$ é o número de características na análise e $\mathrm{L}$ é a matriz diagonal de autovalores de $\mathrm{S}_{\mathrm{A}}$.

Para análise de Fatores (AF), o modelo $\mathrm{MC}$ foi decomposto, como segue: $\mathrm{y}=\mathrm{Xb}+\mathrm{Z} * \mathrm{c}+\mathrm{Zs}+e$, em que: $Z^{*}=Z\left(I_{N A} \ddot{A} G_{m}\right)$, com $\operatorname{Var}(c)=I_{m} \ddot{A}$ e $\operatorname{Var}(\mathrm{s})=\mathrm{YÄA}$, sendo que $\mathrm{G}$ é a matriz de cargas fatoriais (ordem k x m); Y é a matriz diagonal (ordem k) das variâncias dos fatores específicos (s); c é o vetor de fatores comuns de tamanho $\mathrm{mN}_{\mathrm{A}}$; $\mathrm{s}$ o vetor de fatores específicos de tamanho $\mathrm{km}$, e $\mathrm{m}$ é o número de fatores comuns ajustados.

As equações de modelos mistos para as análises de componentes principais $\mathrm{e}$ análises de fatores foram descritas por MEYER \& KIRKPATRICK (2005) e MEYER (2009). Os componentes de (co)variâncias foram estimados pelo método da máxima verossimilhança restrita (REML), utilizando o programa Wombat (MEYER, 2006).

Os modelos foram comparados pelo logaritmo da função de verossimilhança (log L), critério de informação de Akaike (AIC), AKAIKE (1973) e critério de informação Bayesiano (BIC), SCHWARZ (1978) e análise gráfica dos parâmetros genéticos estimados. O AIC e o BIC permitem a comparação entre os modelos não hierárquicos e penalizam aqueles com maior número de parâmetros, como segue: $\mathrm{AIC}=-2 \log \mathrm{L}+2 \mathrm{p}$ e $\mathrm{BIC}=-2 \log \mathrm{L}+$ $\mathrm{p} \log (\mathrm{N}-\mathrm{r})$, em que: $\mathrm{p}=$ número de parâmetros do modelo, $\mathrm{N}=$ número total de observações, $\mathrm{r}=$ posto da matriz de incidência dos efeitos fixos no modelo e log $\mathrm{L}=$ logaritmo da função de máxima verossimilhança restrita.

\section{RESULTADOS E DISCUSSÃO}

Os valores dos critérios estatísticos para escolha de modelos, Log L, AIC e BIC, apresentados na tabela 2, aumentaram com o aumento do número de parâmetros até o $\mathrm{CP} 4$ e AF4, decrescendo para os modelos CP5 e AF5,

Tabela 2 - Número de parâmetros (np), função de verossimilhança (LogL), critério de informação de Akaike (AIC), critério de informação Bayesiano de Schwarz (BIC) e proporção da variância genética aditiva $(\lambda$, em \%), explicada pelos três primeiros autovalores para os modelos de posto completo $(\mathrm{MC})$ e posto reduzido $\left(\mathrm{CP}_{\mathrm{n}}\right.$ e $\left.\mathrm{AF}_{\mathrm{n}}\right)$ e valores de $\mathrm{LogL}$, AIC e BIC como desvios em relação ao modelo multi-característica de posto completo.

\begin{tabular}{|c|c|c|c|c|c|c|c|}
\hline Modelos $^{1}$ & $\mathrm{np}$ & $\log L$ & AIC & BIC & $\lambda_{1}(\%)$ & $\lambda_{2}(\%)$ & $\lambda_{3}(\%)$ \\
\hline CP1 & 65 & -20754 & -20664 & $-20382,09$ & 100,00 & 0,00 & 0,00 \\
\hline $\mathrm{CP} 2$ & 74 & -7662 & -7590 & $-7364,47$ & 75,88 & 24,12 & 0,00 \\
\hline CP3 & 82 & -874 & -818 & $-642,59$ & 64,26 & 20,38 & 15,37 \\
\hline $\mathrm{CP} 4$ & 89 & 54 & 96 & 227,55 & 61,74 & 19,50 & 14,66 \\
\hline CP5 & 95 & 52 & 82 & 175,96 & 61,80 & 19,49 & 14,57 \\
\hline $\mathrm{MC}$ & 110 & 0.00 & 0.00 & 0,00 & 61,72 & 19,57 & 14,45 \\
\hline $\mathrm{AF} 1$ & 75 & -16676 & -16606 & $-16386,74$ & 100,00 & 0,00 & 0,00 \\
\hline $\mathrm{AF} 2$ & 84 & -7662 & -7610 & $-7447,12$ & 75,88 & 24,12 & 0,00 \\
\hline AF3 & 92 & -874 & -838 & $-725,23$ & 64,24 & 20,42 & 15,34 \\
\hline AF4 & 99 & 52 & 74 & 142,91 & 61,71 & 19,54 & 14,63 \\
\hline AF5 & 105 & 52 & 62 & 93,32 & 61,76 & 19,54 & 14,58 \\
\hline
\end{tabular}

${ }^{1} \mathrm{CPn}=$ modelo ajustando $\mathrm{n}$ componentes principais; $\mathrm{MC}=$ modelo multi-característica de posto completo; $\mathrm{AFn}=$ modelo de análise de fatores com $\mathrm{n}$ fatores. Valores em negrito indicam o melhor modelo, com base em AIC e BIC.

Ciência Rural, v.45, n.6, jun, 2015. 
indicando que o modelo de componentes principais CP4 e o modelo de análise de fatores AF4 foram mais eficientes. Ao comparar o modelo CP4 com o AF4, observa-se que o primeiro resultou em melhores valores de Log L, AIC e BIC. O modelo CP4 estimou 21 parâmetros a menos que o MC e 10 a menos que o AF4, o que diminui a demanda computacional. De acordo com MEYER (2007), quando se utiliza um número reduzido de componentes principais, uma pequena parte da variação genética não é particionada, sendo incluída no componente residual. Por outro lado, nos modelos de fatores, grande parte dessa variação é incorporada nas variâncias específicas.

O número de componentes principais indicado neste trabalho foi superior ao relatado por BIGNARDI et al. (2010) e PEREIRA et al. (2013) para a raça Gir e BIGNARDI et al. (2012) para a raça Holandesa. Esses autores relataram que apenas dois componentes principais foram suficientes para modelar a estrutura de covariâncias genéticas entre as produções de leite no dia do controle.

A soma dos três primeiros autovalores, referentes à matriz de covariância genética aditiva, obtidos pelo modelo MC, explicaram 95,74\% da variação genética total entre os animais $(11=61,72$; $12=19,57 ; 13=14,45)$. Resultado semelhante foi observado para o modelo CP4 (Tabela 2), sendo os três primeiros autovalores responsáveis por explicar $95,90 \%$ da variação genética total $(11=61,74 ; 12=19,50 ; 13=14,60)$. MARDIA et al. (1997) sugeriram que os primeiros componentes principais devem explicar, pelo menos, $90 \%$ da variação total.
As variâncias genéticas aditivas, residuais e herdabilidades foram praticamente iguais para $\mathrm{MC}$, CP4 e AF4 (Tabela 3). As estimativas de variância genética das PLDC obtidas para o modelo MC foram levemente inferiores às obtidas pelos modelos $\mathrm{CP} 4 \mathrm{eAF} 4$, para quase todo período da lactação (Tabela 3), sendo as maiores estimativas obtidas para PL1 $(15,38)$ e PL10 $(20,97)$. Ao contrário do observado para as variâncias genéticas, a menor estimativa de variância residual foi observada no final da lactação (PL10). A magnitude das estimativas das variâncias residuais para a maioria dos controles mensais de produção sugere a existência de grandes diferenças de meio ambiente entre os rebanhos incluídos no estudo, principalmente na primeira metade da lactação (até PL6).

As herdabilidades estimadas para as dez produções de leite no dia do controle, utilizando os modelos MC, CP4 e AF4, foram similares e variaram de 0,06 (PL6) a 0,65 (PL10), com erro padrão variando de 0,002 a 0,06 , sendo os maiores erros observados nas PLs 9 e 10, provavelmente, devido ao menor número de observações verificado nestes meses de produção (Tabela 1). Nos três modelos, as estimativas de herdabilidade foram altas nas extremidades da curva (Tabela 3). Estes resultados não concordam com o relatado por VAN VLECK \& HENDERSON (1961), os quais afirmaram que os primeiros e os últimos meses da lactação são mais sujeitos às variações temporárias de meio ambiente e que as produções do meio da lactação são mais influenciadas pelas diferenças genéticas e permanentes de meio, existentes entre as vacas. A alta herdabilidade estimada para o PL10 pode ser devida também ao menor número de

Tabela 3 - Estimativas de componentes de variância e de coeficientes de herdabilidade pelo modelo multi-característica (MC), modelo ajustando os quatro primeiros componentes principais (CP4) e modelo ajustando quatro fatores (AF4), para produção de leite no mês do controle (PL1, ..., PL10).

\begin{tabular}{|c|c|c|c|c|c|c|c|c|c|}
\hline & \multicolumn{3}{|c|}{---------Variância genética aditiva--------- } & \multicolumn{3}{|c|}{--------Variância residual--------- } & \multicolumn{3}{|c|}{-----------Herdabilidade---------. } \\
\hline & $\mathrm{MC}$ & $\mathrm{CP} 4$ & AF4 & $\mathrm{MC}$ & $\mathrm{CP} 4$ & AF4 & $\mathrm{MC}$ & $\mathrm{CP} 4$ & AF4 \\
\hline PL1 & 15,38 & 15,75 & 15,73 & 27,76 & 27,44 & 27,46 & 0,36 & 0,36 & 0,36 \\
\hline PL2 & 2,76 & 2,78 & 2,78 & 35,13 & 35,13 & 35,13 & 0,07 & 0,07 & 0,07 \\
\hline PL3 & 3,74 & 3,73 & 3,73 & 34,01 & 34,02 & 34,02 & 0,10 & 0,10 & 0,10 \\
\hline PL4 & 10,16 & 10,28 & 10,29 & 26,03 & 25,96 & 25,96 & 0,28 & 0,28 & 0,28 \\
\hline PL5 & 4,48 & 4,49 & 4,49 & 29,10 & 29,10 & 29,10 & 0,13 & 0,13 & 0,13 \\
\hline PL6 & 1,83 & 1,84 & 1,84 & 27,32 & 27,32 & 27,32 & 0,06 & 0,06 & 0,06 \\
\hline PL7 & 6,57 & 6,62 & 6,63 & 20,09 & 20,06 & 20,06 & 0,25 & 0,25 & 0,25 \\
\hline PL8 & 6,19 & 6,25 & 6,24 & 20,83 & 20,79 & 20,80 & 0,23 & 0,23 & 0,23 \\
\hline PL9 & 11,73 & 11,91 & 11,92 & 17,82 & 17,68 & 17,68 & 0,40 & 0,40 & 0,40 \\
\hline PL10 & 20,97 & 21,19 & 21,24 & 11,32 & 11,16 & 11,12 & 0,65 & 0,65 & 0,65 \\
\hline
\end{tabular}

Ciência Rural, v.45, n.6, jun, 2015. 
observações disponíveis para esse controle, o que pode ter superestimado a variância genética aditiva presente na população para esta característica. Estimativas discordantes das obtidas neste estudo foram relatadas por BIGNARDI et al. (2008), os quais encontraram estimativas das herdabilidades para as PLDC, por análises unicaracterística, entre 0,07 e 0,19 , sendo o maior valor obtido para o terceiro controle.

As estimativas de correlações genéticas obtidas pelo MC foram iguais às obtidas pelo CP4 e AF4 e variaram de 0,05 a 0,94 , sendo menores que as relatadas por MELO et al. (2005), que variaram de 0,64 a 1,00. As estimativas de correlações fenotípicas entre os controles mensais de lactação foram positivas e variaram de 0,17 a 0,71 , semelhantes às encontradas por BIGNARDI et al. (2008) que estimaram valores variando de 0,17 a 0,68 , para a mesma raça.

\section{CONCLUSÃO}

A estrutura de covariâncias genéticas entre as produções de leite no dia do controle pode ser ajustada utilizando um modelo de posto reduzido, contendo quatro componentes principais ou quatro fatores, pois diminui consideravelmente o número de parâmetros a serem estimados, sendo uma opção a ser adotada nas avaliações genéticas.

\section{COMITÊ DE ÉTICA E BIOSSEGURANÇA}

Nós autores do artigo intitulado "Parâmetros genéticos para produção de leite no dia do controle de vacas da raça Holandesa utilizando modelos de análises de fatores e componentes principais" declaramos, para os devidos fins, que o projeto que deu origem aos dados deste trabalho não foi submetido para avaliação ao Comitê de Ética da Universidade Federal de Santa Maria, mas estamos cientes do conteúdo das resoluções do Conselho Nacional de Controle de Experimentação Animal - CONCEA < http://www. mct.gov.br/index.php/content/view/310553.html> caso envolva animais.

Dessa forma, os autores assumem total responsabilidade pelos dados apresentados e estão disponíveis para possíveis questionamentos, caso venham a ser requeridos pelos órgãos competentes.

\section{AGRADECIMENTOS}

Agradecimento à Coordenação de Aperfeiçoamento de Pessoal de Nível Superior (CAPES), pela concessão de bolsa de estudo à Mariana de Almeida Dornelles.

\section{REFERÊNCIAS}

AKAIKE, H.v Information theory and an extension of the maximum likelihood principle. In: INTERNATIONAL SYMPOSIUM ON
INFORMATION THEORY, 2., 1973, Budapest. Proceedings... Budapest: Academia iKiado, 1973. p.267-281.

BIGNARDI, A.B. et al. Análises multi-características da produção de leite no dia do controle de vacas da raça Gir utilizando componentes principais. In: SIMPÓSIO BRASILEIRO DE MELHORAMENTO ANIMAL, 8., 2010, Maringá, PR. Anais... Maringá: SBMA, 2010. CD-ROM.

BIGNARDI, A.B. et al. Modelos de dimensão finita para a estimação de parâmetros genéticos para a produção de leite de primeiras lactações de vacas da raça Holandesa. Ciência Rural, v.38, n.6, p.1705-1710, 2008. Disponível em: <http://dx.doi. org/10.1590/S0103-84782008000600033>. Acesso em: 01 jan. 2013. doi: $10.1590 / \mathrm{S} 0103-84782008000600033$.

BIGNARDI, A.B. et al. Short communication: principal components and factor analytic models for test-day milk yield in Brazilian Holstein cattle. Journal of Dairy Science, v.95, n.4, p.2157-2164, 2012. Disponível em: <http://dx.doi.org/10.3168/ jds.2011-4494>. Acesso em: 10 jun. 2013. doi: 10.3168/ jds.2011-4494

KIRKPATRICK, M.; MEYER, K. Direct estimation of genetic principal components: simplified analysis of complex phenotypes. Genetics, v.168, p.2295-2306, 2004. Disponível em: <http:// dx.doi.org/10.1534/genetics.104.029181>. Acesso em: 20 nov. 2013. doi: 10.1534/genetics.104.029181.

MADSEN, P. et al. Reduced rank estimation of (co)variance components for international evaluation using AI-REML. Interbull Bull, v.25, p.46-50, 2000.

MARDIA, K.V. Multivariate analysis. 6.ed. London: Academic, 1997. 518p. Disponível em: <http://dx.doi. org/0.1186/1297-9686-43-33>. Acesso em: 18 ago. 2013. doi: $10.1186 / 1297-9686-43-33$

MELO, C.M.R. et al. Parâmetros genéticos para as produções de leite no dia do controle e da primeira lactação de vacas da raça Holandesa. Revista Brasileira de Zootecnia, v.34, n.3, p.796806, 2005. Disponível em: <http://dx.doi.org/10.1590/S151635982005000300011>. Acesso em: 30 dez. 2013. doi: 10.1590/ S1516-35982005000300011.

MEYER, K.; KIRKPATRICK, M. Restricted maximum likelihood estimation of genetic principal components and smoothed covariance matrices. Genetics Selection Evolution, v.35, p.1-30, 2005. Disponível em: <http://dx.doi. org/10.1186/1297-9686-37-1-1>. Acesso em 12 jan. 2013. doi: $10.1186 / 1297-9686-37-1-1$.

MEYER, K. Multivariate analyses of carcass traits for Angus cattle fitting reduced rank and factor analytic models. Journal Animal Breeding Genetics, v.124, p.50-63, 2007. Disponível em: $<\mathrm{http}$ ://dx.doi.org/10.1111/j.1439-0388.2007.00637.x>. Acesso em: 30 jul. 2013. doi: 10.1111/j.1439-0388.2007.00637.x.

MEYER, K. WOMBAT - A tool for mixed model analyses in quantitative genetics by REML. Journal of Zhejiang University Science B, v.8, n.11, p.815-821, 2007. Disponível em: <http:// dx.doi.org/10.1631/jzus.2007.B0815>. Acesso em: 20 jan. 2013. doi: 10.1631/jzus.2007.B0815.

MEYER, K. Factor-analytic models for genotype x environment type problems and structured covariance matrices. Genetics 
Selection Evolution, v.41, p.21-32, 2009. Disponível em: <http:// dx.doi.org/10.1186/1297-9686-41-21>. Acesso em: 12 jan. 2013. doi: 10.1186/1297-9686-41-21.

PEREIRA, R.J. et al. Comparison of factor -analytic and reduced rank models for test-day milk yield in Gyr dairy cattle (Bos indicus). Genetic and Molecular Research, v.12, n.3, p.40364045, 2013. Disponível em: <http://dx.doi.org/10.4238/2013>. Acesso em: 12 fev. 2014. doi: 10.4238/2013.

SCHWARZ, G. Estimating the dimensional of a model. Annals of Statistics, Hayward, v. 6, n. 2, p. 461-464, 1978.

SWALVE, H.H. The effect of test day models on the estimation of genetic parameters and breeding values for dairy yield traits. Journal of Dairy Science, v.78, n.4, p.929-938, 1995. Disponível em: $\quad<$ http://dx.doi.org/10.3168/jds.S0022-0302(95)76708-X>. Acesso em: 28 set. 2013. doi: 10.3168/jds.S0022-0302(95)76708-X.

THOMPSON, R. et al. A sparse implementation of the average information algorithm for factor analytic and reduced rank variance models. Australian New Zealand Journal Statistics, v.45, p.445-459, 2003. Disponível em: $<\mathrm{http}$ ://dx.doi.org/10.1111/1467842X.00297>. Acesso em: 15 jan. 2014. doi: 10.1111/1467$842 X .00297$.

VAN VLECK, L.D.; HENDERSON, C.R. Estimates of genetic parameters of some functions of part lactation milk records. Journal of Dairy Science, v.44, n.6, p.1073, 1961. Disponível em: <http://dx.doi.org/10.3168/jds.S0022-0302(61)898585>. Acesso em: 20 fev. 2014. DOI: 10.3168/jds.S00220302(61)89858-5. 